\title{
SENTIDOS ATRIBUÍDOS À SOBRECARGA DE TRABALHO POR RESIDENTES DE GINECOLOGIA E OBSTETRÍCIA E SUA INFLUÊNCIA NO PROFISSIONALISMO MÉDICO
}

\author{
Elaine Saraiva Feitosa', Ana Maria Fontenelle Catrib1, Aline Veras Morais Brilhante', Sammya \\ Bezerra Maia e Holanda Moura ${ }^{1}$, Sílvia de Melo Cunha ${ }^{1}$ e Christina César Praça Brasil ${ }^{1}$ \\ 1 Universidade de Fortaleza, Brasil. elainesfeitosa@gmail.com catrib@unifor.br; alineveras@unifor.br; \\ sammyabezerra@uol.com.br; silviamelo87@gmail.com; cpraca@unifor.br
}

\begin{abstract}
Resumo. A residência médica $(\mathrm{RM})$ é a melhor forma de capacitação do médico em serviço. Discute-se o profissionalismo médico em Ginecologia e Obstetrícia (GO) devido às constantes crises na relação médico-paciente. A sobrecarga de trabalho evidencia-se nesse contexto por suas influências negativas no desempenho profissional. Objetivou-se conhecer os sentidos atribuídos à sobrecarga de trabalho pelos residentes de GO e sua influência no profissionalismo médico. Estudo exploratório e qualitativo, com a realização de grupos focais com 21 residentes de GO de quatro programas de RM do estado do Ceará, Brasil. Analisaram-se os dados por meio da análise de conteúdo na modalidade temática e a interpretação dos achados respaldou-se no Interacionismo Simbólico. Os resultados mostram que a sobrecarga de trabalho está impregnada de sentidos que geram prejuízos à saúde mental dos residentes de GO, prejudicando o profissionalismo médico. Observa-se, também, a tomada de consciência em grupo para o enfrentamento do adoecimento e da medicalização, além da busca pela promoção da saúde. Por fim, considera-se que o conhecimento dos sentidos atribuídos pelos residentes à sobrecarga de trabalho auxilia na ampliação do olhar dos coordenadores da RM, fazendo com que repensem as estratégias de formação e ofereçam medidas para a melhoria das condições de trabalho.
\end{abstract}

Palavras-chave: Profissionalismo Médico; Residência Médica; Ginecologia; Obstetrícia; Condições De Trabalho

\section{MEANINGS ATTRIBUTED TO WORK OVERLOAD BY RESIDENTS OF GYNECOLOGY AND OBSTETRICS AND ITS INFLUENCE ON MEDICAL PROFESSIONALISM}

\begin{abstract}
Medical residency (MR) is the best way to train doctors in service. Medical professionalism in Gynecology and Obstetrics (GO) is discussed due to the constant crises in the doctor-patient relationship. Work overload is evidenced in this context by its negative influences on professional performance. The objective was to know the meanings attributed to work overload by residents of $\mathrm{GO}$ and its influence on medical professionalism. An exploratory and qualitative study, with focus groups conducted with $21 \mathrm{GO}$ residents from four MR programs in the state of Ceará, Brazil. The data were analyzed through content analysis in the thematic modality and the interpretation of the findings was supported by Symbolic Interactionism. The results show that work overload is impregnated with meanings that generate damage to the mental health of GO residents, impairing medical professionalism. It is also observed the awareness of the group to face illness and medicalization, in addition to the search for health promotion. Finally, it is considered that the knowledge of the meanings attributed by residents to work overload helps to broaden the view of MR coordinators, making them (re)think training strategies and offer measures to improve working conditions.
\end{abstract}

Keywords: Medical Professionalism; Medical Residency; Gynecology; Obstetrics; Working Conditions

\section{INTRODUÇÃO}

A Residência Médica(RM) é a modalidade de treinamento em serviço considerada como a melhor forma de capacitação do médico (Brasil, 1977). Nesse contexto, além da abordagem técnica da especialidade a que se destina, a RM também objetiva formar o médico no 
desenvolvimento de habilidades e atitudes, as quais são imprescindíveis para o bom exercício da profissão (Rego, 2012).

A capacitação em serviço oportuniza ao futuro especialista conviver com a realidade e as demandas específicas da área de atuação. No contexto da Ginecologia e Obstetrícia (GO), o ambiente de trabalho é marcado por longas jornadas de atuação; dedicação intensiva a alguns casos, a exemplo de trabalhos de parto e risco materno-fetal; ansiedade das pacientes e familiares; dentre outros aspectos que podem comprometer física e emocionalmente os residentes e os médicos do serviço (Rodrigues et al., 2018; Rua, Body, Marret, \& Ouldamer, 2015) Assim, para que haja o bom direcionamento das ações e relações de trabalho, além do cumprimento dos princípios éticos, o profissionalismo é peça chave no processo de formação dos residentes.

O profissionalismo médico permanece como um construto sem homogeneidade entre as especialidades médicas (Feitosa et al., 2019) que envolve múltiplas dimensões - pessoais, sociais , institucionais e interpessoais (Consorti, Notarangelo, Potasso, \& Toscano, 2012), sendo essencial na relação do médico com a sociedade (Cruess, 2006). O American Board of Internal Medicine (ABIM) definiu profissionalismo como o conjunto de responsabilidades profissionais que incluem atualização constante, honestidade, confidencialidade, a boa relação com pacientes, familiares e membros da equipe de saúde, empenhando-se na melhoria constante da qualidade do atendimento (ABIM Foundation. American Board of Internal Medicine, 2002). Na RM, conforme o Conselho de Acreditação Médica de Pósgraduação (ACGME) os estudantes devem demonstrar compaixão, sensibilidade, honestidade, respeito à diversidade, gerenciamento de conflitos, assegurando os interesses dos pacientes acima dos seus (The Accreditation Council for Graduate Medical Education, 2017).

O profissionalismo médico ganhou destaque na última década pelas constantes crises sociais na relação médico-paciente. A carta sobre o profissionalismo médico publicada em 2002 pelas sociedades europeias e americanas, subscrita por vários programas de RM, orientou a atuação do médico juntamente com as contribuições do ACGME nas estratégias de ensino e avaliação do profissionalismo (Meakins, 2003; The Accreditation Council for Graduate Medical Education, 2017). A despeito das recomendações, observa-se que em algumas especialidades existe uma demanda crescente de processos éticos e disciplinares, 
notadamente, na área de Ginecologia e Obstetrícia (Silva, Rodrigues e Rodrigues, \& Bernardes, 2015).

Os problemas de profissionalismo médico são objeto de estudo há muitas décadas, diante da relevância do tema e da preocupação com a formação de novos profissionais. De acordo com Rego (2012), a inclusão de discussões sobre o Código de Ética nos programas de formação não garante o pleno ensino do profissionalismo, em decorrência da sua complexidade. Para o autor, é necessário que todos os envolvidos no processo de formação - professores, alunos, residentes e profissionais do serviço - tenham a consciência de que o profissionalismo é parte integrante da convivência social que firma a profissão na sociedade, sendo uma das dimensões mais importantes para o exercício profissional.

A sobrecarga de trabalho, verificada por meio das longas jornadas e da multiplicidade de atribuições no contexto profissional, figura como um dos elementos que trazem comprometimento a saúde física e mental de muitos residentes de GO. Essa problemática pode comprometer as relações interpessoais, além da qualidade técnica e humana do atendimento prestado à população.

Conforme Fabichak, Silva-Júnior, \& Morrone, (2014, p. 83), o relato de sobrecarga de trabalho durante a residência médica de GO pode estar relacionado "à grande demanda de pacientes e reduzida equipe para suprir o atendimento. $O$ excesso de trabalho, tal qual ocorre em serviços de atendimento emergencial, pode causar gradativa exaustão emocional, física e/ou mental".

Este estudo justifica-se pela necessidade de verificar, nos programas de RM em Ginecologia e Obstetrícia, as percepções que os residentes têm da sobrecarga de trabalho e sua influência na vida profissional. Acredita-se que esse conhecimento poderá favorecer a reflexão, a reestruturação dos programas e a redução dos lapsos na atuação com pacientes e familiares.

Nessa perspectiva, objetivou-se conhecer os sentidos atribuídos à sobrecarga de trabalho pelos residentes de GO e sua influência no profissionalismo médico.

\section{METODOLOGIA}

Trata-se de um estudo exploratório, com abordagem qualitativa, realizado em Fortaleza, Ceará, Brasil, em setembro de 2019. A pesquisa qualitativa é bastante pertinente para esta 
investigação por favorecer a descrição, categorização, interpretação e compreensão dos fatos que abordam os significados, motivos, aspirações, crenças, valores, percepções e atitudes - elementos determinantes das relações sociais, dos processos e dos fenômenos (Minayo, 2014). Nessa perspectiva, "o foco da investigação recai sobre a palavra que expressa a fala, a percepção cotidiana dos sujeitos, seja nas relações sociais, afetivas e técnicas, seja nos discursos intelectuais, burocráticos e políticos" (Minayo \& Sanches, 1993, p. 245).

Participaram do estudo 21 residentes de GO, de quatro programas de RM do estado do Ceará, Brasil. A Residência Médica de Ginecologia e Obstetrícia do município de Fortaleza é composta pelos programas da Secretaria Municipal de Saúde (SMS), do Hospital Geral César Cals, do Hospital Geral de Fortaleza e do Programa de Residência Médica (RM) da Maternidade Escola Assis Chateaubriand. Juntos, compreendem 95 médicos residentes.

Para compor a amostra deste estudo, o convite foi feito por e-mail para todos os residentes dos programas de GO descritos nesta sessão. As listas de e-mails foram fornecidas, mediante autorização prévia, pelos referidos programas e pela Sociedade Cearense de Ginecologia e Obstetrícia (SOCEGO). Do universo total (95) de residentes, 21 concordaram em participar da pesquisa e compareceram na data de realização dos grupos focais.

No que concerne aos instrumentos e estratégias para a coleta de dados, utilizaram-se Grupos Focais (GF). Segundo Backes et al. (2011), no campo da pesquisa qualitativa, o GF é um tipo de entrevista em grupo que permite um processo dinâmico de coleta de dados, a partir da interação grupal, promovendo a ampla problematização sobre um fenômeno específico (Backes, Colomé, Erdmann, \& Lunardi, 2011). Tem como base a tendência humana de formar opiniões e atitudes na interação social com outros sujeitos, além de explorar suas concepções e experiências.

O grupo focal possibilitou captar a percepção dos residentes de GO sobre o construto de profissionalismo e o processo de ensino-aprendizagem; porém questões relacionadas a sobrecarga de trabalho e as suas implicações no profissionalismo médico emergiram de forma espontânea nos relatos. Assim, este trabalho constitui o recorte de um estudo mais amplo e merece ser explorado de forma específica, diante da sua relevância para a formação dos residentes. 
Dois grupos focais foram realizados na mesma data, no mês de setembro de 2019, em salas cedidas pela SOCEGO. Cada grupo foi conduzido por uma facilitadora (uma em cada sala), um relator (um em cada sala) e um observador (um em cada sala). Os grupos foram organizados com 10 e 11 participantes, respectivamente, para contemplar os 21 residentes que atenderam ao convite para participar do estudo, os quais foram inicialmente esclarecidos sobre os objetivos da pesquisa e assinaram o Termo de Consentimento Livre e Esclarecido.

Após a coleta dos dados sociodemográficos, por meio de um instrumento estruturado, a partir do qual se coletaram informações referentes a sexo, idade, procedência, tempo no programa de residência, procedeu-se à realização do grupo focal baseada em questões norteadoras que versavam sobre: O que significa profissionalismo para você? Na sua visão, como acontece o ensino do profissionalismo na formação do residente? Como acontece o processo de formação em profissionalismo na sua residência? Como você busca o aperfeiçoamento em profissionalismo? De que modo estão inseridas as estratégias de ensino de profissionalismo na matriz de competências? Como você percebe as contribuições do ensino de profissionalismo na mudança de atitude profissional? O que pode ser feito para aprimorar o ensino do profissionalismo na residência de Ginecologia e Obstetrícia?

A coleta de dados foi gravada e registrada em cada grupo, tendo o primeiro grupo duração de 57 minutos e o segundo, duração de uma hora e treze minutos. Os dados, após coletados, foram ouvidos na íntegra pelas pesquisadoras e transcritos. Em seguida, fez-se a leitura em profundidade das transcrições e iniciou-se a organização e análise do material.

Para o tratamento dos dados, utilizou-se análise de conteúdo na modalidade temática (Minayo, Deslandes, Cruz Neto, \& Gomes, 2013), que diz respeito a técnicas que proporcionam replicabilidade e inferências sobre um contexto. Para isso, a partir da leitura do texto transcrito percorreram-se as seguintes etapas: pré-análise, exploração do material e tratamento dos resultados - inferência e interpretação (Maria Cecília de Souza Minayo, 2014).

No intuito de identificar as áreas temáticas e compreender o objeto de estudo e as suas nuanças, foram empregados, de forma interpretativa, os conceitos do Interacionismo Simbólico. Nesse contexto, as relações sociais exercem papel preponderante na formação de conceitos e na modelagem de atitudes a partir dos sentidos e significados provenientes 
das interações em comunidade. Blumer (1969) afirmou que os sujeitos que vivem em comunidade agem de acordo com os significados atribuídos às coisas e situações a partir das relações estabelecidas no convívio social. O autor caracteriza e sistematiza essas premissas da seguinte forma:

O ser humano age em relação às coisas, na base dos sentidos que elas têm para ele. O sentido dessas coisas é derivado, ou surge, da interação social. Esses sentidos são manipulados e modificados através de um processo interpretativo usado pela pessoa ao tratar as coisas que ela encontra nas interrelações sociais (Blumer, 1969, p. 2).

A vida em sociedade, os grupos e organizações pressupõem interações sociais contínuas e cujas ações resultam de respostas de um em relação ao outro. Os sentidos atribuídos, sua definição e interpretação dos objetos são produtos das relações sociais. Os grupos focais foram denominados "Grupo A" e "Grupo B" e os participantes pela letra "R", que remete a "residente", seguida dos números 1 a 21 , no intuito de preservar o sigilo e o anonimato. Dessa forma, R1 significa "residente 1" e assim sucessivamente.

A partir dos dados coletados, emergiram duas temáticas, as quais serão apresentadas e discutidas na sessão subsequente.

O projeto que originou este estudo foi aprovado pelo Comitê de Ética e Pesquisa em Seres Humanos (COÉTICA), obtendo aprovação sob o número 2.038.755, por estar em conformidade com princípios éticos das pesquisas com seres humanos.

\section{RESULTADOS E DISCUSSÃO}

A partir das análises realizadas neste estudo, verificou-se que os sentidos atribuídos à sobrecarga de trabalho por residentes de Ginecologia e Obstetrícia influenciam direta ou indiretamente o profissionalismo médico, fazendo emergir as seguintes temáticas: impactos da sobrecarga de trabalho na saúde mental e estratégias de enfrentamento. Esses sentidos estão permeados por ações e interpretações, de acordo com o que preconiza o Interacionismo Simbólico (Blumer, 1969), os quais podem ser melhor compreendidos por meio dos núcleos de sentido que se associam a cada uma das temáticas.

\subsection{Impactos da sobrecarga de trabalho na saúde mental}

As interpretações dos residentes sobre os impactos da sobrecarga de trabalho na saúde mental refletem-se nos seguintes aspectos: descaso com o autocuidado; dificuldades na 
relação médico-paciente e comportamento agressivo; e falta de suporte à saúde mental dos residentes e medicalização para amenizar o sofrimento mental.

Os participantes referiram que a sobrecarga de trabalho leva ao autocuidado comprometido, o que os faz descuidarem da alimentação, do controle de peso, da rotina de atividade física e das relações interpessoais. Os depoimentos a seguir trazem o descaso com 0 autocuidado e os seus impactos no profissionalismo como foco da discussão:

[...] Esta semana, eu estava discutindo com o meu grupo de R2 [residentes do segundo ano] sobre uma das coisas que a gente como médico tem mais dificuldade que é o autocuidado. Então [...] é muito difícil você cuidar [do outro], se você não se cuidar, principalmente na residência [...]. Então, o autocuidado e o tempo de a gente dar uma pausa para ir a uma terapia, ir a uma academia..., a gente nunca vai conseguir refletir, pensar sobre a gente, se a gente não pára... Tem simplesmente momentos que a gente não pára. A gente está discutindo, ah... e eu estou refletindo. Honestamente, tem horas que ninguém consegue refletir nada! A gente parece mais uma máquina! (R13)

[...] a questão da alimentação é péssima. Quantas de vocês comem bem [pergunta feita olhando para os colegas do grupo focal que também são residentes]? Tem dias que passa batido [a pessoa não se alimenta] e assim suga tanto que você esquece de comer. (R16)

Os residentes mencionam que o descaso com a saúde é inerente ao profissional de Medicina e que essa realidade piora no período da RM. De fato, estudos demonstram que durante a RM o residente tem uma carga horária de trabalho superior a 60 horas semanais, o que é preconizado pelos programas, perfazendo uma média de 68,7 horas semanais. Essa realidade decorre da baixa remuneração da bolsa de residência médica que obriga os jovens a buscarem atividades remuneradas fora do horário, emendando, muitas vezes, jornadas de trabalho e comprometendo o autocuidado. Nesse sentido, as queixas mais frequentes são: cansaço mental $(95,8 \%)$ e pouco tempo para si mesmo $(91,6 \%)$, dentre outras (Fabichak et al., 2014).

Na mesma linha, um estudo qualitativo realizado com 21 residentes de uma escola médica no Canadá revelou a conexão que os estudantes fazem entre duvidar de si mesmos e o medo de fraquejar, diante da dificuldade de demonstrar empatia em situações dramáticas. $O$ discurso de um deles admite que se alguém se encontra fisicamente exausto, dificilmente continuará a prover atendimento de qualidade aos pacientes(Phillips \& Dalgarno, 2017).

A importância do autocuidado também foi levantada em um estudo envolvendo 100 estudantes provenientes de 10 escolas médicas americanas, os quais, em suas falas, incluem as habilidades de autocuidado e autorreflexão como prioridades profissionais 
essenciais, além de pontuarem que devem ser ensinados a se auto empoderar para que possam melhor ajudar os seus pacientes e a honrarem a si próprios para que possam honrar outrem (Rabow, Wrubel, \& Remen, 2009).

Com o decorrer do tempo, a falta de cuidado leva ao desgaste físico e emocional, comprometendo o desempenho das atividades profissionais, o que, muitas vezes, prejudica a relação médico-paciente, levando alguns residentes a perceberem-se agressivos e cometendo lapsos de profissionalismo, como mostram os trechos a seguir, que versam sobre as dificuldades na relação médico-paciente e comportamento agressivo:

[...] Às vezes eu me pego no meio do atendimento, porque tem ainda várias fichas [pessoas] para atender, e me pego assim no meio da anamnese dizendo à paciente o meu nome ... Era para eu ter feito isso no começo da consulta, mas eu vi seis fichas lá para atender e eu estou sozinha no Serviço de Pronto Atendimento, então ficou mecanizado e já fui perguntando a queixa e depois que eu lembrei de me apresentar a paciente. (R8)

[...] E você começa a ser agressiva nas palavras quando você está estressada. Você começa a não pensar direito como você está agindo, porque você está em Burnout e o Burnout médico é difícil de ser entendido, porque ele [o médico] só aceita que está em Burnout quando já está em grau extremo. Diferente de outros profissionais que eu acho que acabam procurando ajuda mais cedo. (R13)

Um estudo de Castelhano (2015) abordou as influências das emoções na percepção do paciente sobre as relações com o médico, sob a perspectiva da psicologia junguiana. Os resultados mostraram que as emoções exercem uma forte influência sobre as atitudes e que os médicos revelam sofrimento diante dos problemas de relacionamento com seus pacientes e colegas; o que os faz referir sobre o elevado nível de estresse ao qual estão sujeitos e a necessidade de um espaço onde possam ser melhor compreendidos e receber suporte emocional para que as emoções possam refletir positivamente nas relações no ambiente de trabalho.

O relato de $\mathrm{R} 8$ evidencia que a sobrecarga de trabalho associada a elevada demanda de pacientes leva a um sofrimento mental dos residentes, o que, muitas vezes, emerge em forma de falhas na comunicação médico-paciente, a exemplo não se identificar no início da consulta. R13, por sua vez, reflete sobre a agressividade gerada pelo estresse decorrente do Burnout, além da dificuldade do médico em reconhecer essa situação.

Outro aspecto bastante destacado pelos participantes deste estudo diz respeito a falta de suporte à saúde mental dos residentes e a medicalização para amenizar o sofrimento mental. Esses aspectos evidenciam-se pelo fato de os residentes sentirem-se desassistidos 
pelos programas de residência no que diz respeito à saúde mental, fazendo com que o nível de estresse e sofrimento mental evoluam e requeiram medicação para amenizar o problema.

[...] Às vezes, eu também acho que não é tratada a saúde mental dos residentes porque a pressão, a sobrecarga, às vezes, tem uma hora que ele explode e aquele residente que está sozinho não tem ninguém para ajudá-lo... Talvez, o coordenador da residência seja a pessoa à qual ele possa recorrer para pedir ajuda. E eu acho também válido trabalhar a saúde mental dos residentes, porque o índice de suicídio nas escolas de residências aumentou. (R4)

[...] Às vezes, não é nem falta de ajuda, porque às vezes a pessoa não consegue pedir ajuda. Talvez as pessoas [...], os staffs, os preceptores, fossem até treinados a observar sinais [de sofrimento dos residentes] porque isso nunca acontece de repente, eles vão evoluindo ao longo do tempo. [...] Talvez se tivesse um treinamento com os staffs para perceberem isso e acolherem esses residentes, talvez facilitaria. (R9)

[...] A questão é que muitas vezes a gente está sozinha. Principalmente no plantão noturno, você fica lá levando a sala de parto sozinha e o teu staff não te dá apoio. Então, se um problema desse acontece, por mais que a gente seja residente, a gente é médico. Então, por mais que tenha alguém para responder com a gente, vamos responder também por algum problema que aconteça. Isso é muito desgastante. (R18)

[...] o índice de Burnout em GO é altíssimo e, na nossa residência, todo ano tem pesquisas. Eu estava espantada com o índice de medicalização dos residentes... É altíssima quantidade de residentes que tomam medicação para dormir, estabilizantes do humor, entre outros. Eu acho que na nossa residência esse quantitativo de pessoas que se medicalizam é perto de $30 \%$. (R13)

Muitos dos sentimentos negativos expressos pelos residentes advêm da falta de apoio e percepção dos preceptores e dos staffs sobre o sofrimento mental dos residentes, causado pela demanda de trabalho à qual estão expostos. Muitos residentes sentem-se sobrecarregados e abandonados à rotina de trabalho, sem contar com uma estrutura de apoio do programa de residência para a prevenção, a identificação e o enfrentamento do problema.

$\mathrm{Na}$ fala de R9, evidencia-se a necessidade de treinamento dos staffs e dos preceptores para que estes percebam os problemas de saúde mental decorrentes da sobrecarga de trabalho que acometem os residente, no sentido de que sejam melhor preparados para auxiliá-los a superar o cansaço, a angústia e evitar prejuízos na postura profissional e nas relações de trabalho. O estudo canadense demonstra a mesma percepção por parte dos estudantes envolvidos, onde estes observam que os preceptores e os supervisores possivelmente 
também não tenham preparo emocional para lhes dar suporte em suas angústias (Phillips \& Dalgarno, 2017).

Para Blumer (1969), as percepções das pessoas que se estabelecem pela interação social e vivência dos contextos facilitam a identificação de problemas, fazendo emergirem soluções, a exemplo da sugestão de uma capacitação para a equipe gestora da residência em GO. Esta alternativa, advinda dos olhos de quem conhece a situação na prática, poderá auxiliar a minimizar o problema e a criar ações que ofereçam suporte aos residentes que, de alguma forma, seja nas palavras, seja nas ações, sinalizem problemas na saúde mental e no profissionalismo.

A participante R13 relata sobre a elevada taxa de frequência de Burnout e a medicalização entre seus pares. Esta fala revela a necessidade de alguns residentes recorrerem a medicamentos para aliviar o sofrimento mental, o cansaço e até buscarem uma melhor qualidade de sono. Esse caminho, entretanto, pode causar danos à saúde física e mental dessa população.

Na compreensão da análise do comportamento profissional, Moreira, Souza, \& Yamaguchi, (2018) apontam a síndrome de Burnout como responsável pelos lapsos profissionais, caracterizada pelo tripé: exaustão emocional (EE), despersonalização (DP) e comprometimento da realização profissional (RP). Por conseguinte, ocorre cansaço, distanciamento dos colegas e baixa empatia. É bem estudado que o cansaço excessivo afeta a relação do médico com seus pacientes, colegas de trabalho e equipe, sendo este um grave problema de saúde pública que merece destaque por atingir com frequência profissionais da saúde sobretudo, de forma crítica, os médicos de várias especialidades como evidenciado nesse estudo (Moreira et al., 2018).

\subsection{Estratégias de Enfrentamento}

Diante dos problemas referidos, os residentes buscam estratégias para enfrentar as situações de dificuldades e sofrimento mental referidas, buscando melhorias na saúde mental, que refletem na boa atuação profissional. Nessa temática, surge a forte ideia de que o amparo do grupo é essencial, a partir do estabelecimento conjunto de metas e da troca de ideias, que funcionam como uma "terapia em grupo", como revela R13.

[...] a gente fez do nosso grupo um lugar para enfrentar os problemas dessa sobrecarga de trabalho [...] Cada um fez metas por escrito de autocuidado. Eu fui para a academia depois de anos, porque senão a 
gente não vai se cuidar nunca! A gente fez as metas por escrito. Entre elas, eu coloquei como outra meta, comer pelo menos uma fruta ao dia. [...] Meu Deus! Quantos de nós tomamos medicações? Quantos? Quantos? E mais ou menos assim o que a gente estava refletindo em grupo, levou-nos a fazer basicamente uma terapia em grupo sobre isso. (R13)

O empoderamento e a sensação de amparo são elementos que surgem nas interpretações dos participantes, ao relatarem o poder das estratégias em grupo para o enfrentamento do sofrimento mental e a busca da melhoria para o exercício profissional. As atividades curriculares complementares e alternativas inclusas para os programas de formação, especialmente relacionadas ao desenvolvimento de técnicas de integração mente-corpo e autocuidado, têm sido apontadas como eficientes na melhora do profissionalismo entre os estudantes em escolas americanas (Elder, Hustedde, Rakel, \& Joyce, 2008).

Nessa perspectiva, a partir da visão de Blumer (1967), verificam-se três premissas básicas do Interacionismo Simbólico que facilitam a compreensão do potencial da interação grupal para a promoção de mudanças nos comportamentos das pessoas. A primeira dessas premissas revela que "O ser humano age com relação às coisas na base dos sentidos que elas têm para ele" (Haguette, 2013, p. 34). A convivência em grupo, principalmente com pessoas que vivenciam situações similares ajuda na significação e na ressignificação de conceitos e ideias, uma vez que a segunda premissa defende que "O sentido destas coisas é derivado, ou surge, da interação social que alguém estabelece com os seus companheiros"(Haguette, 2013,p.34).Dessa forma, "Estes sentidos são manipulados e modificados através de um processo interpretativo usado pela pessoa ao tratar as coisas que ela encontra." (Haguette, 2013, p.34).

O relato de R13 reforça claramente a ideia de que a ação, muitas vezes, deriva de uma interação social recíproca, sendo esta capaz de modificar-se progressivamente, contando com o apoio das relações interpessoais e das especificidades das situações (Lopes \& Jorge, 2005). No contexto da sobrecarga de trabalho, os residentes, ao pactuarem metas de melhorias de qualidade de vida em grupo, amenizam física e emocionalmente o fardo que a sobrecarga do trabalho Ihes trazem, fazendo com que comparem esta ação a uma "terapia em grupo".

Nas interações grupais, os residentes, no contexto em estudo, apreendem os significados da sobrecarga de trabalho, agem conforme as indicações que fazem para si no aprendizado mútuo e interpretam a necessidade de mudança da realidade, levando à adoção de 
estratégias de enfrentamento, a partir do estabelecimento de metas claras e por escrito para que possam acompanhar o seu cumprimento. $\mathrm{O}$ ambiente de aprendizagem influencia $\mathrm{O}$ modo de pensar e agir dos sujeitos que vivem em comunidade, os quais agem de acordo com os significados atribuídos às coisas nas interações com os outros, evidenciado nas estratégias de enfrentamento do grupo ao ressignificar as interações simbólicas do adoecimento físico e mental.

\section{CONCLUSÃO}

O estudo qualitativo, com base no Interacionismo Simbólico, traz contribuições importantes na perspectiva da pesquisa qualitativa, por aprofundar o olhar na subjetividade e no contexto dos programas de residência médica em GO e mostrar, a partir das percepções dos residentes, os sentidos atribuídos à sobrecarga de trabalho e sua influência no profissionalismo médico, o que se desdobra em dois eixos principais: os impactos da sobrecarga de trabalho na saúde mental e as estratégias de enfrentamento adotadas por eles. Isto mostra a necessidade de uma avaliação dos programas de RM e a verificação de alternativas para melhorar as condições de trabalho e qualidade de vida pessoal e profissional dos residentes.

A sobrecarga de trabalho leva a um processo de adoecimento mental que prejudica o profissionalismo médico, principalmente ao comprometer o autocuidado, levar a comportamentos agressivos e prejudicar as relações interpessoais. Observou-se que as atitudes dos residentes não são notadas pelos preceptores e supervisores dos programas, o que requer capacitação e sensibilização para que possam melhor acolhê-los e auxiliá-los a lidar com as situações que geram desconforto e adoecimento. Ademais, a ampliação do suporte à saúde mental dos residentes pode reduzir a medicalização para amenizar o sofrimento mental.

Nesse contexto, o significado atribuído ao apoio dos pares, por meio das interações em grupo, deve ser fortemente considerado pelos programas de residência, uma vez que geram ressignificações para a busca de alternativas de enfrentamento, as quais amenizam o sofrimento gerado pela sobrecarga de trabalho, melhoram o profissionalismo e ajudam na promoção da saúde. 


\section{REFERÊNCIAS}

ABIM Foundation. American Board of Internal Medicine. (2002). Medical professionalism in the new millennium: a physician charter. Annals of Internal Medicine, 136(3), 243.

Backes, D. S., Colomé, J. S., Erdmann, R. H., \& Lunardi, V. L. (2011). Grupo focal como técnica de coleta e análise de dados em pesquisas qualitativas. Mundo Da Saude, 35(4), 438-442. https://doi.org/10.1017/CBO9781107415324.004

Blumer, H. (1969). Symbolic interactionism: Perspective and method. 1969. Englewood Cliffs: Prentice Hall.

BRASIL. (1977). Decreto No 80.281, de 5 de setembro de 1977. Regulamenta a Residência Médica, cria a Comissão Nacional de Residência Médica e dá outras providências. Retrieved March 28, 2019, from http://www.planalto.gov.br/ccivil_03/decreto/1970-1979/D80281.htm

Castelhano, L. M. (2015). As emoções do médico na relação com o paciente: uma abordagem da psicologia junguiana.

Consorti, F., Notarangelo, M., Potasso, L., \& Toscano, E. (2012). Developing professionalism in Italian medical students: an educational framework. Advances in Medical Education and Practice, 3, 55.

Cruess, S. R. (2006). Professionalism and medicine's social contract with society. Clinical Orthopaedics and Related Research, (449), 170-176. https://doi.org/10.1097/01.blo.0000229275.66570.97

Elder, W. G., Hustedde, C., Rakel, D., \& Joyce, J. (2008). CAM curriculum activities to enhance professionalism training in medical schools. Complementary Health Practice Review, 13(2), 127-133.

Fabichak, C., Silva-Júnior, J. S., \& Morrone, L. C. (2014). Síndrome de burnout em médicos residentes e preditores organizacionais do trabalho. Rev Bras Med Trab, 12(2), 79-84.

Feitosa, E. S., Brilhante, A. V. M., Cunha, S. de M., Sá, R. B., Nunes, R. R., Carneiro, M. A., ... Catrib, A. M. F. (2019). Professionalism in the Training of Medical Specialists: an Integrative Literature Review. Revista Brasileira de Educação Médica, 43(1), 692-699.

Haguette, T. M. F. (2013). Metodologias qualitativas na sociologia. (Vozes, Ed.) (14th ed.). Petrópolis.

Lopes, C. H. A. de F., \& Jorge, M. S. B. (2005). Interaccionismo simbólico y la posibilidad para el cuidar interactivo de la enfermería. Revista Da Escola de Enfermagem Da USP, 39(1), 103-108.

Meakins, J. L. (2003). Medical professionalism in the new millenium. Journal of the American College of Surgeons, 196(1), 113-114. Retrieved from http://www.ncbi.nlm.nih.gov/pubmed/12517562

Minayo, M. C. D. S., Deslandes, S. F., Cruz Neto, O., \& Gomes, R. (2001). (2013). Pesquisa social: teoria, método e criatividade. (33rd ed.). Petrópolis: Vozes.

Minayo, Maria Cecilia de S, \& Sanches, O. (1993). Quantitativo-qualitativo: oposição ou complementaridade? Cadernos de Saúde Pública, 9(3), 237-248.

Minayo, Maria Cecília de Souza. (2014). O Desafio do Conhecimento: Pesquisa Qualitativa em Saúde (14a.). São PAULO: Hulcitec.

Moreira, H. D. A., Souza, K. N. De, \& Yamaguchi, M. U. (2018). Síndrome de Burnout em médicos: uma revisão sistemática. Revista Brasileira de Saúde Ocupacional, 43, 1-11. https://doi.org/10.1590/23176369000013316

Phillips, S. P., \& Dalgarno, N. (2017). Professionalism, professionalization, expertise and compassion: a qualitative study of medical residents. BMC Medical Education, 17(1), 1-7. https://doi.org/10.1186/s12909017-0864-9 
Rabow, M. W., Wrubel, J., \& Remen, R. N. (2009). Promise of professionalism: personal mission statements among a national cohort of medical students. The Annals of Family Medicine, 7(4), 336-342.

Rego, S. (2012). O Profissionalismo e a Formação Médica. Revista Brasileira de Educação Médica, pp. 445-446.

Rodrigues, H., Cobucci, R., Oliveira, A., Cabral, J. V., Medeiros, L., Gurgel, K., ... Gonçalves, A. K. (2018). Burnout syndrome among medical residents: A systematic review and meta-analysis. PloS One, 13(11).

Rua, C., Body, G., Marret, H., \& Ouldamer, L. (2015). Prévalence du syndrome d'épuisement professionnel parmi les internes de gynécologie-obstétrique et facteurs associés. Journal de Gynécologie Obstétrique et Biologie de La Reproduction, 44(1), 83-87.

Silva, F., Rodrigues e Rodrigues, M., \& Bernardes, J. (2015). Disciplinary actions in gynecology and obstetrics in the north of Portugal from year 2008 to 2012. Acta Medica Portuguesa, 28(2), 194-204.

The Accreditation Council for Graduate Medical Education. (2017). Common Program Requirements Section VI with Background and Intent. 